\title{
Dynamical Model of Synchronous Impact Electromagnetic Drive
}

\author{
V.Yu. Neyman \\ Novosibirsk State Technical University \\ Novosibirsk, Russian Federation \\ e-mail: nv.nstu@ngs.ru
}

\author{
L.A Neyman \\ Novosibirsk State Technical University \\ Novosibirsk, Russian Federation \\ e-mail: neyman31@gmal.com
}

\begin{abstract}
Mechanisms and units with reciprocated impulse electromagnetic drives are widely used in many industrial technological processes for material plastic deformation and disruption. With respect to modern energy saving requirements, low-frequency impact synchronous electromagnetic machines attract attention as their impact pulse frequency is equal to or multiple of single-phase source frequency. The performed research relevance is explained by the need to improve electromagnetic compatibility of the electric drive powered by the industrial single-phase source. It is necessary to study epy electromechanical energy conversion process during the machine operating cycle. The two-inductor synchronous electromagnetic machine with head free running-out in the driving stroke inductor powered by the $50 \mathrm{~Hz}$ single-phase voltage source is considered. The research methods are based on the energy balance of the electromechanical system and its components for the impact unit total operating cycle. With respect to the interaction between all impact unit elements when the head impact mass is accelerated by the magnetic field generated by the system of two inductors, the energy conversion process based on the electromechanical system energy balance is considered for the total operating cycle. The operating cycle implementation in the two-inductor synchronous electromagnetic machine with free running-out permits to reduce current amplitude and to suppress electromagnetic machine influence on mains in comparison with the known operating cycle. The operating cycle with head free running-out in the driving stroke inductor of the synchronous two-inductor impact electromagnetic machine improves electromagnetic compatibility, if the machine is powered by a single-phase voltage.
\end{abstract}

Keywords - mathematical and dynamical models; mechatronic model; electromagnetic impact drive; mechanical oscillating system; dynamical characteristics, machine operating cycle.

\section{INTRODUCTION}

Power electromagnetic impulse systems became widely used as executive reciprocated electric drives including different electromagnetic machines, vibration sources, press equipment, electrically operated hand tools, etc. [1-5].

The head impact mass motion simplifies the unit structure and kinematic scheme and permits one to improve energy, economic and ecology indicators relative to other machines and units [6].
Electromagnetic machines design methods are wellknown. Their improvement goes on [7-8]. Low-frequency synchronous impact electromagnetic machines are distinguished by better energy indicators. Their impact pulses frequency is equal to or multiple of power source frequency [9].

Electric energy conversion to effective work is impulsive. Then impact energy is limited by allowable pulse power of the $50 \mathrm{~Hz}$ source. To reduce electric drive influence on the power line a new operating cycle is needed. The proposed cycle is implemented by the mechatronic module of the impact twoinductor synchronous electromagnetic machine.

The research purpose is the development of a mathematical model of the two-inductor impact electromagnetic machine with head free running-out. The model should provide the capability of making the detailed analysis of the electromagnetic and electromechanical processes more impartially reflecting the considered system parameters interconnections in different operating modes.

\section{TWO-INDUCTOR SYNCHRONOUS IMPACT ELECTROMAGENTIC MACHINE MECHATRONIC MODULE MATHEMATICAL MODEL}

Fig. 1 shows one of the possible variants of the twoinductor synchronous electromagnetic machine.

The new operating cycle is implemented by the system of the two inductors 1, 2 inside the magnetic cores 3, 4. Each inductor provides acceleration of the impact mass of the head 5 by electromagnetic forces in two directions. When the head 5 is reciprocating it interacts periodically with the tool 6 and the stopping and reversing return spring 7 . The press force $f_{\text {pr }}$ provides the stable link between the impact unit and the tool when periodic impact pulses are generated with respect to the interaction with the deformed medium 8 .

The complete operating cycle corresponds to three power line voltage periods to provide the head impact synchronous frequency $n_{\mathrm{im}}$ and operating cycle $t_{\mathrm{c}}$.

$$
\text { Hence, } n_{\mathrm{im}}=\frac{60 f}{2 p}=1000 \mathrm{~min}^{-1}, t_{\mathrm{c}}=\frac{2 \partial}{f}=0.06 \mathrm{~s} \text {, }
$$

where $2 p=3$ is the number of voltage periods, $f=50 \mathrm{~Hz}$. 
Both inductors are used two times during the operating cycle. The head accumulates necessary kinetic energy because of applying four voltage pulses to the inductors.

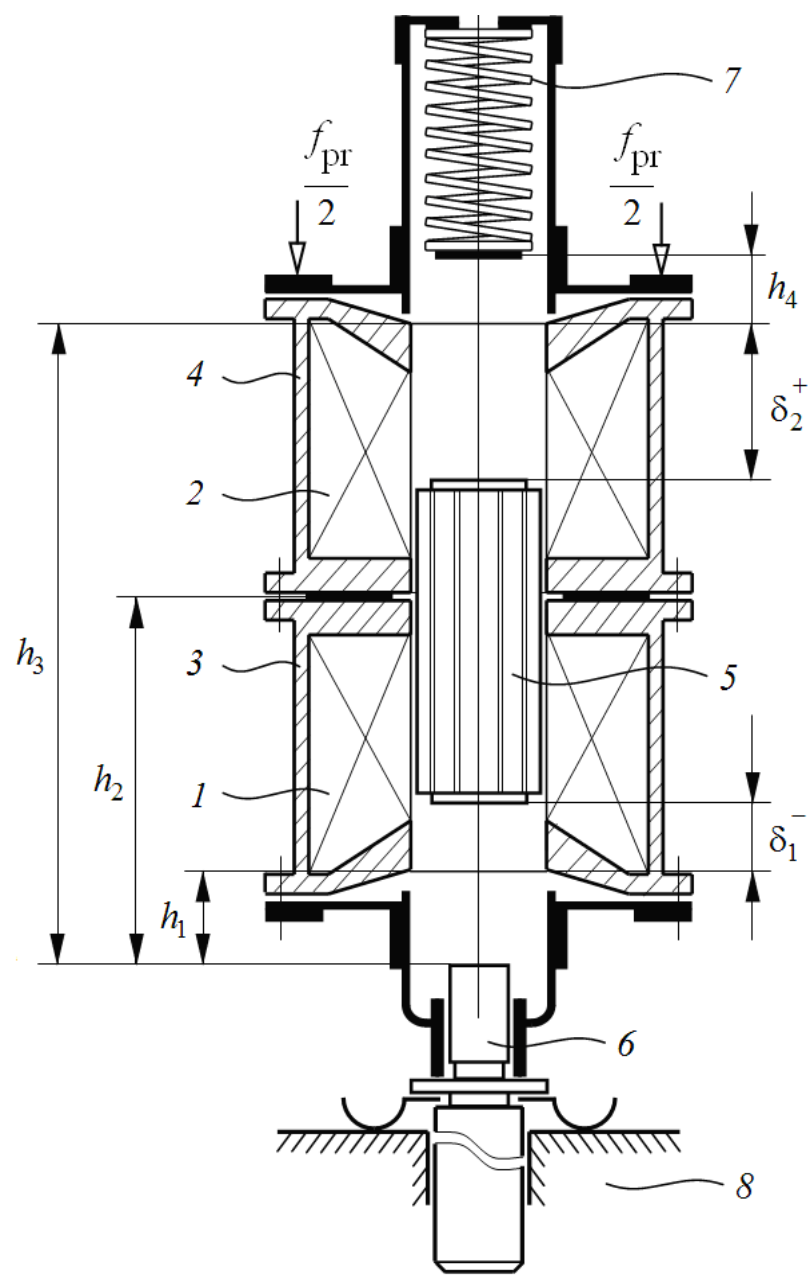

Fig. 1. Electromagnetic impact unit

The impact unit mechanical subsystem and magnetic subsystem interaction is described by the electromagnetic force dependences $f_{\mathrm{em} 1}=f\left(i_{1}, x_{1}\right)$ and $f_{\mathrm{em} 2}=f\left(i_{2}, x_{1}\right)$, where $i_{1}, i_{2}$ are the currents in the inductors, $x_{1}$ is the head position coordinate. The magnetic subsystem and electric subsystem interaction is described by the flux dependences $\psi_{1}=f\left(i_{1}, x_{1}\right)$ and $\psi_{2}=f\left(i_{2}, x_{1}\right)$. Generally magnetic and electric subsystems interaction is described by the electrical balance equations:

$$
\begin{aligned}
& u_{1}(t)=i_{1} r_{1}+\frac{d \psi_{1}\left(i_{1}, x_{1}\right)}{d t} \\
& u_{2}(t)=i_{2} r_{2}+\frac{d \psi_{2}\left(i_{2}, x_{1}\right)}{d t},
\end{aligned}
$$

where $u_{1}(t), u_{2}(t)$ are the inductors windings voltages, $r_{1}$, $r_{2}$ are the inductor circuits active resistances.

With respect to the interactions between elements (Fig. 1) and generalized forces, corresponding to potential energy, dissipation energy and external actions, Fig. 2 shows the design dynamic scheme of the electromechanical impact unit mechanical part.

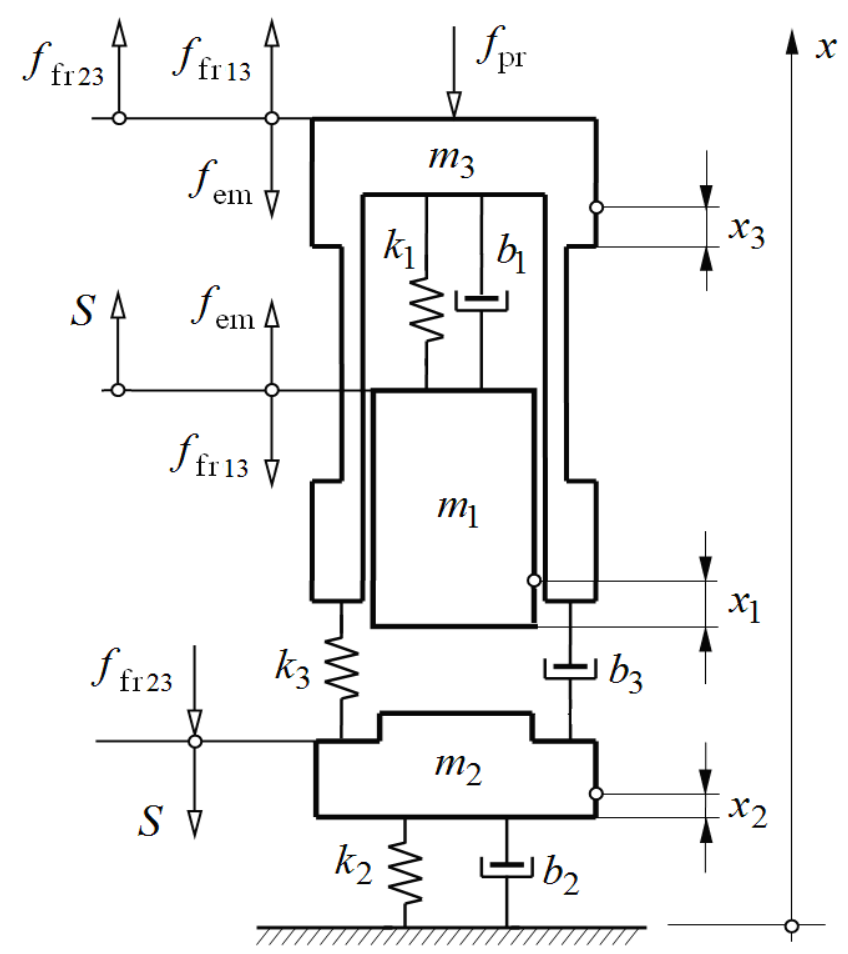

Fig. 2.Mechanical system design dynamic scheme

The mechanical system motion equation is derived from the Lagrange equation of the second type:

$$
\frac{d}{d t}\left[\frac{\partial T}{\partial \dot{x}_{i}}\right]-\frac{\partial T}{\partial x_{i}}=-\frac{\partial U}{\partial x_{i}}-\frac{\partial F}{\partial \dot{x}_{i}}+Q_{i}, i=1,2, \ldots, N
$$

where $T$ is the system kinetic energy; $U$ is the system potential energy, $F$ is the system dissipative function or the Rayleigh function; $\frac{\partial U}{\partial x_{i}}, \frac{\partial F}{\partial \dot{x}_{i}}, Q_{i}$ are generalized elastic forces, friction forces and external forces for the $i$-th generalized coordinate, $x_{i}$ is the $i$-th generalized coordinate, $\dot{x}_{i}$ is the $i$-th generalized velocity; $N$ is the mechanical system freedom degrees number $(N=3)$.

Further the magnitudes in (3) are expressed.

The kinetic energy of the mechanical system for directly moving masses is: 


$$
T=\frac{m_{1} \dot{x}_{1}^{2}}{2}+\frac{m_{2} \dot{x}_{2}^{2}}{2}+\frac{m_{3} \dot{x}_{3}^{2}}{2} .
$$

The mechanical system spring linkages potential energy is:

$$
U=\frac{k_{1}\left(x_{1}-x_{3}\right)^{2}}{2}+\frac{k_{2} x_{2}^{2}}{2}+\frac{k_{3}\left(x_{3}-x_{2}\right)^{2}}{2},
$$

where $k_{1}, k_{2}$ and $k_{3}$ are the spring linkages static stiffness factors.

The Rayleigh dissipative function is:

$$
F=\frac{b_{1}\left(\dot{x}_{1}-\dot{x}_{3}\right)^{2}}{2}+\frac{b_{2} \dot{x}_{2}^{2}}{2}+\frac{b_{3}\left(\dot{x}_{3}-\dot{x}_{2}\right)^{2}}{2}
$$

where $b_{1}, b_{2}$ and $b_{3}$ are the spring linkages viscous friction factors.

The generalized external forces and friction forces corresponding to the generalized coordinated are:

$$
\begin{aligned}
& Q_{x_{1}}=f_{\mathrm{em}}\left(i, x_{1}\right)-f_{\mathrm{fr}_{13}} \operatorname{sign} \dot{x}_{1} ; \\
& Q_{x_{2}}=-f_{\mathrm{fr}_{23}} \operatorname{sign} \dot{x}_{2} ; \\
& Q_{x_{3}}=-f_{\mathrm{em}}\left(i, x_{1}\right)+\left(f_{\mathrm{fr}_{13}}+f_{\mathrm{fr}_{23}}\right) \operatorname{sign} \dot{x}_{3}-f_{\mathrm{pr}},
\end{aligned}
$$

where $f_{\mathrm{em}}\left(i, x_{1}\right)=f_{\mathrm{em} 1}\left(i_{1}, x_{1}\right)+f_{\mathrm{em} 2}\left(i_{2}, x_{1}\right)$ is the driving electromagnetic force of the first and the second inductor, $f_{\mathrm{fr}}, f_{\mathrm{fr} 23}$ are the dry sliding friction forces; $f_{\mathrm{pr}}$ is the constant force which is pressing the impact unit;

- partial derivatives of kinetic energy and time derivatives:

$$
\begin{aligned}
& \frac{d}{d t}\left(\frac{\partial T}{\partial \dot{x}_{1}}\right)=m_{1} \ddot{x}_{1}, \quad \frac{\partial T}{\partial x_{1}}=0 ; \\
& \frac{d}{d t}\left(\frac{\partial T}{\partial \dot{x}_{2}}\right)=m_{2} \ddot{x}_{2}, \quad \frac{\partial T}{\partial x_{2}}=0 ; \\
& \frac{d}{d t}\left(\frac{\partial T}{\partial \dot{x}_{3}}\right)=m_{3} \ddot{x}_{3}, \quad \frac{\partial T}{\partial x_{3}}=0,
\end{aligned}
$$

- partial derivatives of potential energy from generalized coordinates:

$$
\frac{\partial U}{\partial x_{1}}=k_{1}\left(x_{1}-x_{3}\right)
$$

$$
\frac{\partial U}{\partial x_{2}}=k_{2} x_{2}-k_{3}\left(x_{3}-x_{2}\right)
$$

$$
\frac{\partial U}{\partial x_{3}}=-k_{1}\left(x_{1}-x_{3}\right)+k_{3}\left(x_{3}-x_{2}\right)
$$

- partial derivatives of the dissipative function by the generalized velocity:

$$
\begin{aligned}
& \frac{\partial F}{\partial \dot{x}_{1}}=b_{1}\left(\dot{x}_{1}-\dot{x}_{3}\right) \\
& \frac{\partial F}{\partial \dot{x}_{2}}=b_{2} \dot{x}_{2}-b_{3}\left(\dot{x}_{1}-\dot{x}_{2}\right) ; \\
& \frac{\partial F}{\partial \dot{x}_{3}}=-b_{1}\left(\dot{x}_{1}-\dot{x}_{3}\right)+b_{3}\left(\dot{x}_{3}-\dot{x}_{2}\right) .
\end{aligned}
$$

Introducing the derived values of the derivatives of the kinetic energy and the generalized forces corresponding to the potential energy, the scattering energies and external influences, into Lagrange's equations, the differential equations of motion of the mechanical system of the model were deduced:

$$
\left\{\begin{aligned}
m_{1} \ddot{x}_{1}= & -k_{1}\left(x_{1}-x_{3}\right)-b_{1}\left(\dot{x}_{1}-\dot{x}_{3}\right)+f_{\mathrm{em}}\left(i, x_{1}\right)+ \\
& -f_{\mathrm{fr}_{13}} \operatorname{sign} \dot{x}_{1} ; \\
m_{2} \ddot{x}_{2}= & -k_{2} x_{2}+k_{3}\left(x_{3}-x_{2}\right)-b_{2} \dot{x}_{2}+b_{3}\left(\dot{x}_{1}-\dot{x}_{2}\right)+ \\
& -f_{\mathrm{fr}_{23}} \operatorname{sign} \dot{x}_{2} ; \\
m_{3} \ddot{x}_{3}= & k_{1}\left(x_{1}-x_{3}\right)-k_{3}\left(x_{3}-x_{2}\right)+b_{1}\left(\dot{x}_{1}-\dot{x}_{3}\right)- \\
& -b_{3}\left(\dot{x}_{3}-\dot{x}_{2}\right)-f_{\mathrm{em}}\left(i, x_{1}\right)+ \\
& +\left(f_{\mathrm{fr}_{13}}+f_{\mathrm{fr}_{12}}\right) \operatorname{sign} \dot{x}_{3}-f_{\mathrm{pr}} .
\end{aligned}\right.
$$

With respect to (1)-(3) and preliminary transformations the oscillatory electromechanical system, the dynamical state mathematical model is described by the following differential equations neglecting the impact interaction:

$$
\begin{aligned}
& u_{1}(t)=i_{1} r_{1}+\frac{d \psi_{1}\left(i_{1}, x_{1}\right)}{d t} ; \\
& u_{2}(t)=i_{2} r_{2}+\frac{d \psi_{2}\left(i_{2}, x_{1}\right)}{d t}
\end{aligned}
$$




$$
\begin{gathered}
m_{1} \frac{d^{2} x_{1}}{d t^{2}}+b_{1}\left(\frac{d x_{1}}{d t}-\frac{d x_{3}}{d t}\right)+k_{1}\left(x_{1}-x_{3}\right)= \\
=f_{\mathrm{em} 1}\left(i_{1}, x_{1}\right)+f_{\mathrm{em} 2}\left(i_{2}, x_{1}\right)-f_{\mathrm{fr} 13} \operatorname{sign} \frac{d x_{1}}{d t} ; \\
m_{2} \frac{d^{2} x_{2}}{d t^{2}}+b_{2} \frac{d x_{2}}{d t^{2}}-b_{3}\left(\frac{d x_{3}}{d t}-\frac{d x_{2}}{d t}\right)+ \\
+k_{2} x_{2}-k_{3}\left(x_{3}-x_{2}\right)=-f_{\mathrm{fr} 23} \operatorname{sign} \frac{d x_{2}}{d t} \\
m_{3} \frac{d^{2} x_{3}}{d t^{2}}-b_{1}\left(\frac{d x_{1}}{d t}-\frac{d x_{3}}{d t}\right)+b_{3}\left(\frac{d x_{3}}{d t}-\frac{d x_{2}}{d t}\right)- \\
-k_{1}\left(x_{1}-x_{3}\right)+k_{3}\left(x_{3}-x_{2}\right)=-f_{\mathrm{em} 1}\left(i_{1}, x_{1}\right)- \\
-f_{\mathrm{em} 2}\left(i_{2}, x_{1}\right)+\left(f_{\mathrm{fr} 13}+f_{\mathrm{fr} 23}\right) \operatorname{sign} \frac{d x_{3}}{d t}-f_{\mathrm{pr}}
\end{gathered}
$$

The limit stops in the mechatronic module do not permit the head to travel out of established limits:

$$
x_{1}(t)=\left\{\begin{array}{l}
0, \text { at } \delta_{1}^{+}=h_{1} ; \\
h_{1}-\delta_{1}^{+}, \text {if } \frac{d x_{1}}{d t}>0, \text { at } x_{1} \leq h_{1} ; \\
h_{3}-\delta_{2}^{+}, \text {if } \frac{d x_{1}}{d t}>0, \text { at } h_{1}<x_{1} \leq h_{2} ; \\
h_{2}+\delta_{2}^{-}, \text {at } h_{2}<x_{1}<h_{3} ; \\
h_{1}+\delta_{1}^{-}, \text {if } \frac{d x_{1}}{d t}<0, \text { at } h_{1} \leq x_{1}<h_{2} ; \\
h_{1}-\delta_{1}^{+}, \text {if } \frac{d x_{1}}{d t}<0, \text { at } x_{1}<h_{1},
\end{array}\right.
$$

where $\delta_{1}^{+}, \delta_{2}^{+}, \delta_{1}^{-}, \delta_{2}^{-}$are the operating air gaps caused by the head position relative to the poles (Fig.1); $h_{1}, h_{2}$ and $h_{3}$ are the fixed coordinates.

If the motion process is considered as the result of superposition of the forced oscillation and periodic impact pulses, then (4)-(9) allow to present the model of the dynamical state of the impact two-inductor synchronous electromagnetic system as the differential equation system:

$$
\begin{aligned}
& u_{1}(t)=i_{1} r_{1}+\frac{d \psi\left(i_{\mu 1}, x_{1}\right)}{d t} ; u_{2}(t)=i_{2} r_{2}+\frac{d \psi\left(i_{\mu 2}, x_{1}\right)}{d t} ; \\
& i_{1}=i_{\mathrm{L} 1}+i_{\mu 1} ; \quad i_{2}=i_{\mathrm{L} 2}+i_{\mu 2} ; \\
& \left(\frac{m_{1} \bar{v}_{1}}{\tau_{\mathrm{p}}}+f_{\mathrm{em} 1}\left(i_{\mu 1}, x_{1}\right)+f_{\mathrm{em} 2}\left(i_{\mu 2}, x_{1}\right)-\right. \\
& \left\{\begin{array}{l}
-f_{\mathrm{fr}} \operatorname{sign} \frac{d x_{1}}{d t}, \text { if } \frac{d x_{1}}{d t}>0 \\
\text { at } 0 \leq x_{1}<h_{3}+h_{4}
\end{array}\right. \\
& m_{1} \frac{d^{2} x_{1}}{d t^{2}}=\left\{\begin{array}{l}
\text { at } 0 \leq x_{1}<h_{3}+h_{4} ; \\
-\tilde{\lambda}_{1}+f_{\mathrm{em} 2}\left(i_{\mu 2}, x_{1}\right)-f_{\mathrm{fr} 13} \operatorname{sign} \frac{d x_{1}}{d t} \\
\text { at } x_{1} \geq h_{3} ; \\
f_{\mathrm{em} 1}\left(i_{\mu 1}, x_{1}\right)+f_{\mathrm{em} 2}\left(i_{\mu 2}, x_{1}\right)- \\
-f_{\mathrm{fr} 13} \operatorname{sign} \frac{d x_{1}}{d t}, \text { if } \frac{d x_{1}}{d t}<0 \\
\text { at } 0 \leq x_{1}<h_{3}+h_{2} ;
\end{array}\right. \\
& m_{2} \frac{d^{2} x_{2}}{d t^{2}}=-\frac{m_{2} \bar{v}_{2}}{\tau_{\mathrm{p}}}-\tilde{\lambda}_{2}+\tilde{\lambda}_{3}-f_{\mathrm{tr}_{23}} \operatorname{sign} \frac{d x_{2}}{d t} ; \\
& m_{3} \frac{d^{2} x_{3}}{d t^{2}}=\left\{\begin{array}{l}
-\tilde{\lambda}_{3}-f_{\text {эм } 1}\left(i_{\mu 1}, x_{1}\right)-f_{\text {эм } 2}\left(i_{\mu 2}, x_{2}\right)+ \\
+\left(f_{\operatorname{tr}_{13}}+f_{\text {tr } 23}\right) \operatorname{sign} \frac{d x_{3}}{d t}-f_{\mathrm{pr}}, \\
\text { at } 0 \leq x_{1}<h_{3}+h_{2} ; \\
\tilde{\lambda}_{1}-\tilde{\lambda}_{3}-f_{\mathrm{em} 2}\left(i_{\mu 2}, x_{1}\right)-f_{\mathrm{fr}_{13}} \operatorname{sign} \frac{d x_{3}}{d t}, \\
\text { at } x_{1} \geq h_{3}+h_{2},
\end{array}\right.
\end{aligned}
$$

where $\tilde{\lambda}_{1}=b_{1}\left(\frac{d x_{1}}{d t}-\frac{d x_{3}}{d t}\right)+k_{1}\left(x_{1}-x_{3}\right) ; \tilde{\lambda}_{2}=b_{2} \frac{d x_{2}}{d t}+k_{2} x_{2}$; $\tilde{\lambda}_{3}=b_{3}\left(\frac{d x_{3}}{d t}-\frac{d x_{2}}{d t}\right)+k_{3}\left(x_{3}-x_{2}\right) ; \tau_{\mathrm{p}}$ is the impact pulse width, $i_{\mathrm{L} 1}, i_{\mathrm{L} 2}$ are the components of the first and the second inductor magnetizing current; $i_{\mu 1}, i_{\mu 2}$ are correspondingly the components of magnetizing currents in the first and the second inductor.

\section{MATHEMATICAL MODEL IMPLEMENTATION}

The derived differential equations system describing the dynamical state of the two-inductor electromagnetic module permits to make the detailed analysis of electromechanical processes in transient and stationary modes. The equations take into account magnetic system non-linearity, impact interaction between inertial masses, mechanical system spring linkages properties and power loss. 
The model was investigated by structured modeling methods in Matlab Simulink.

Flux linkage and electromagnetic force were calculated in the magnetic field simulation programs FEMM, ELCUT or ANSYS.

Fig. 3 presents the simulation results of impact unit switching on process (Fig. 1).

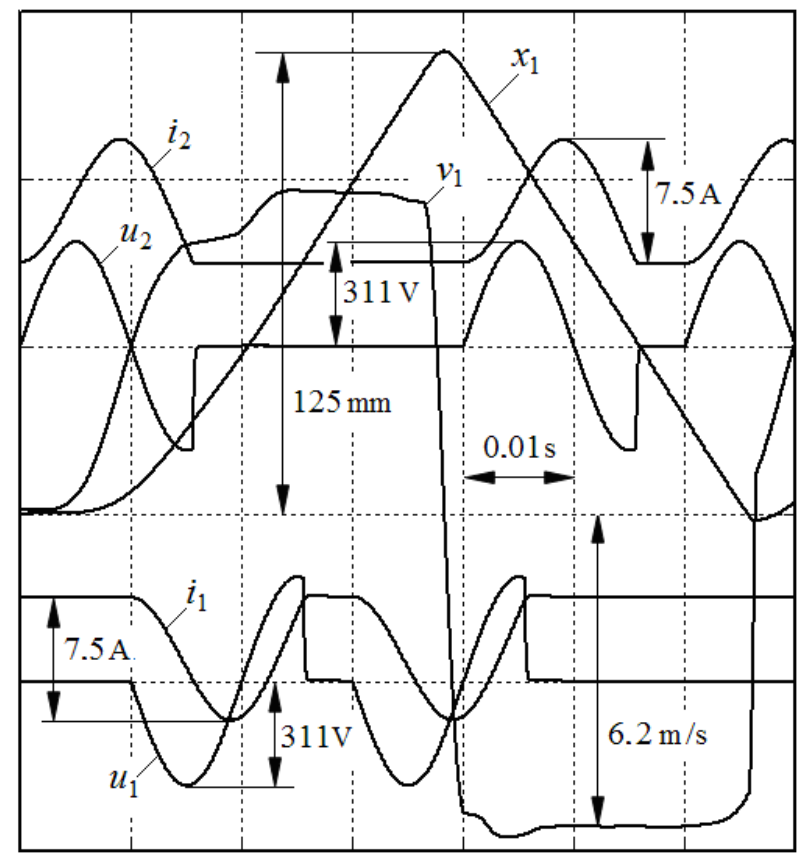

Fig. 3. Switching-on process time diagram

The initial data is $m_{1}=0.32 \mathrm{~kg}, \quad m_{2}=0.36 \mathrm{~kg}$, $m_{3}=4.8 \mathrm{~kg}, \quad k_{1}=2 \cdot 10^{3} \mathrm{~N} / \mathrm{m}, \quad b_{1}=8 \mathrm{~N} \cdot \mathrm{s} / \mathrm{m}$, $k_{2}=2 \cdot 10^{6} \mathrm{~N} / \mathrm{m}, b_{2}=0, k_{3}=16 \cdot 10^{3} \mathrm{~N} / \mathrm{M}, b_{3}=45 \mathrm{~N} \cdot \mathrm{s} / \mathrm{m}$, $f_{\text {fr13 }}=4 \mathrm{~N}, f_{\text {fr23 }}=10 \mathrm{~N}$.

The impact unit press force is $f_{\mathrm{pr}}=350 \mathrm{~N}$. The magnetic core is made of the electrical steel 1212. The head is made of the structural steel $40 \mathrm{XH}$. The inductor windings are made of copper conductor with the diameter $d_{\mathrm{c}}=1.04 \mathrm{~mm}$, the windings number is $w_{1}=w_{2}=1525$, active resistance $r_{1}=r_{2}=12.8 \Omega$. The power supply voltage is equal to $220 \mathrm{~V}$.

The impact unit transient process is no more than one operating cycle and is equal to $0.06 \mathrm{~s}$ (Fig. 3).

In comparison with the single-inductor variant of the mechatronic module, the two-inductor variant provides double head impact energy increase, when current pulses are kept at the previous level.

\section{CONCLUSION}

The mathematical model of the two-inductor synchronous electromagnetic drive mechatronic module dynamics has been developed with respect to the new operating cycle. The model provides capabilities for the detailed analysis in different modes when periodical impact force pulses are generated and interact with deformed medium.

The model important feature is capability of taking into account interconnected electromechanical processes with a big set of input and output variables with respect to materials magnetic characteristics non-linearity, inertial masses mobility degree and spring linkages properties and power loss.

Simulation results approve effectiveness of the new operating cycle providing improvement of electromagnetic compatibility of a single-phase industrial power source and electromagnetic impact drive.

\section{References}

[1] V.N. Oparin, B.F. Simonov, "Nonlinear deformation-wave processes in the vibrational oil geotechnologies," Journal of Mining Science, vol. 46, Num. 2, pp. 95-112, 2010.

[2] V.P. Pevchev, "The superexitation and efficiency relation in a shortstroke pulsed electromagnetic motor of a seismic source", Journal of Mining Science, vol. 46, Num. 6, pp. 656-665, 2010.

[3] Y.N. Zhuravlyov, S.G. Matcevich, F.G. Kochevin, "Low-frequency electrodynamic vibrator with magnetically suspended movable part" Proc. of the 4th Intern. Conf. on Motion and Vibration Control, vol. 3, pp. 1063-1067, 1998.

[4] B.F. Simonov, V.Y. Neyman, A.S. Shabanov, "New conception of an electromagnetic drive for a vibration source in hole" The 18 international conference of young specialists on micro/nanotechnologies and electron devices, EDM 2017: proc., Altai, Erlagol, 29 June - 3 July 2017, Novosibirsk: NSTU, pp. 507-510, 2017.

[5] V.P. Pevchev, "Science of mining machines the superexcitation and efficiency relation in a short-stroke pulsed electromagnetic motor of a seismic source", Journal of Mining Science, vol. 46, Num. 6., pp. 656$665,2010$.

[6] G.G. Ugarov, K.M Usanov, V.Y. Neiman, "Operating Cycle of an electromagnetic percussion machine with storage of magnetic energy during the no-load period" Journal of Mining Sciences, vol. 33. Num. 3. pp. 253-257, 1997.

[7] A.A. Tatevosyan, A.S. Tatevosyan, "Calculation of magnetic system of the magnetoelectric machines" Dynamics of Systems, Mechanisms and Machines, Dynamics 2014 Proceedings, p. 7005698, 2014

[8] S.N. Kuchankov, E.M. Timoshenko, "Nonsteady thermal conditions of electromagnetic motors in shock-type equipment", Journal of Mining Science, vol. 34, Num 2., pp. 148-152, 1998

[9] L.A. Neyman, V.Y. Neyman, K.A. Obukhov, "New method of the synchronous vibratory electromagnetic machine mechatronic module control" The 18 international conference of young specialists on micro/nanotechnologies and electron devices, EDM 2017: proc., Altai, Erlagol, 29 June - 3 July 2017. - Novosibirsk: NSTU, pp. 516-519, 2017. 